TRANSACTIONS OF THE

AMERICAN MATHEMATICAL SOCIETY

Volume 311, Number I, January 1989

\title{
EXCESSIVE MEASURES AND THE EXISTENCE OF RIGHT SEMIGROUPS AND PROCESSES
}

\section{J. STEFFENS}

\begin{abstract}
Given a resolvent $\left(U_{\alpha}\right)$ on a Lusin space $(E, \mathfrak{E})$, the paper gives necessary and sufficient conditions in terms of the excessive measures that ensure the existence of a right process, resp. a right continuous semigroup, on $(E, \mathcal{E})$ with resolvent $\left(U_{(x}\right)$. Furthermore, a notion of nonbranch points with respect to $\left(U_{\alpha}\right)$ is introduced-also in terms of the excessive measures-and various characterizations are given. They show, in particular, the equivalence of this definition with those introduced and discussed by Engelbert and Wittmann.
\end{abstract}

\section{INTRODUCTION}

It is a classical question when a given substochastic resolvent on a measurable space corresponds to a right Markov process or to a right continuous semigroup on that space. Classical answers have been given in the context of (locally) compact spaces for the existence of Feller semigroups and Hunt processes (see, e.g., $[2,5,15])$. Extensions of these classical results to more general settings have been formulated by Engelbert [7, 8]. The most general method of construction of processes so far is the Ray (-Knight) compactification originally introduced by Ray [19]; it provides a Ray semigroup and a Ray process on an enlarged compact state space (see, e.g., [4, 11, 21]). In order to obtain an answer to the initial question via that construction one has to make sure that the Ray semigroup (resp. process) can be restricted to the original space. A sufficient condition is, of course, that the original space coincides with the set of nonbranch points of the Ray semigroup, but this is not necessary.

This article presents characterizations for both the existence of a right continuous semigroup and the existence of a right process corresponding to a given resolvent, by means of conditions on the excessive measures. It seems that in all approaches so far the set of excessive measures has not been considered; the reason may be that interest in properties of excessive measures as opposed to excessive functions has been renewed only recently (see, e.g., $[6,12,13,10])$.

Given a right process with semigroup $\left(P_{t}\right)$ in the sense of [11], the following three facts about the set of excessive measures are well known:

(0.1) Equal measure potentials possess equal charges.

Received by the editors October 7, 1987.

1980 Mathematics Subject Classification (1985 Revision). Primary 60J35, 60J45. 
(0.2) Any excessive measure simply dominated by a measure potential is itself a potential.

(0.3) Any purely excessive measure $m$ can uniquely be decomposed as the integral $m=\int \eta_{t} d t$ of an entrance law $\left(\eta_{t}\right)_{t>0}$ with respect to $\left(P_{t}\right)$.

Proofs of (0.1) and (0.2) can be found in the paper [12] by Getoor and Glover. As to result (0.3), mentioned already by Dynkin [6], several different proofs have been published recently, by Fitzsimmons and Maisonneuve [10], Getoor and Glover [14], Janssen [16], and again Fitzsimmons [9]. The first one by Fitzsimmons and Maisonneuve was purely probabilistic and uses the existence of the stationary process; Fitzsimmons later gave a very nice direct and simple proof using result $(0.2)$. Janssen, furthermore, proved a more general result than (0.3) where he did not assume the existence of a right process, but only of a semigroup on a $U$-space with a resolvent satisfying $(0.1)$.

Result (0.2) states that given a right process the potentials with respect to the simple (in potential theory usually called natural) order form a solid subcone of the cone of all excessive measures. From this the analogous property (0.4) with respect to the specific order follows immediately-a fact that has already been proved by Meyer [18]:

(0.4) Any excessive measure specifically dominated by a measure potential is itself a potential.

The setup for the main part of this paper is the following:

$\left(U_{\alpha}\right)_{\alpha>0}$ denotes a substochastic resolvent with proper potential kernel $U$ on a Lusin space $(E, \mathfrak{E})$. We assume that the $\mathfrak{E}$ measurable $\left(U_{\alpha}\right)$-excessive functions generate the $\sigma$-algebra $\mathfrak{E}$ and that the function 1 is excessive.

Our aim is to show that the properties $(0.1)$ through $(0.3)$ provide also sufficient conditions for the existence of a right process or a right continuous semigroup corresponding to the given resolvent $\left(U_{\alpha}\right)$. Along with these results a new definition for a nonbranch point with respect to the resolvent $\left(U_{\alpha}\right)$ in terms of the excessive measures is studied. Previous definitions (for resolvents on measurable spaces satisfying even more general conditions than stated in (0.5)) were introduced by Engelbert [7] and Wittmann [22]. They turn out to coincide with ours within the given setup.

We say, a point $x \in E$ is a nonbranch point for $\left(U_{\alpha}\right)$ if the measure potential $\varepsilon_{x} U$ lies on an extreme ray in the convex cone of excessive measures with respect to $\left(U_{\alpha}\right)$.

It turns out that given hypotheses $(0.5)$, the extremality of point charges, i.e., property $(0.6)$ for all points in $E$, is equivalent with properties $(0.1)$ and $(0.4)$ on the one hand and with the infimum-stability of the $\left(U_{\alpha}\right)$-excessive functions 
on the other hand, and furthermore with the property that the differences of bounded excessive functions form an algebra.

The two main results are the following:

Under the hypotheses $(0.5)$, properties $(0.1)$ and $(0.2)$ for $\left(U_{\alpha}\right)$ are equivalent with the existence of a (transient) Borel right process with resolvent $\left(U_{\alpha}\right)$.

(0.8) Under the hypotheses $(0.5)$, property (0.3) with an entrance law with respect to $\left(U_{\alpha}\right)$ that charges only the nonbranch points of $\left(U_{\alpha}\right)$ is equivalent with the existence of a right continuous semigroup with resolvent $\left(U_{\alpha}\right)$.

To state the result $(0.8)$, first of all, a proper definition of an entrance law with respect to a resolvent is required. Then what has to be ensured is that the entrances laws corresponding to the measures $U(x$,$) of the potential kernel$ form a semigroup of kernels as desired. Using properties of the set of nonbranch points, the proof is straightforward. More interesting and more surprising than (0.8) appears to be result (0.7). Its proof uses a Ray compactification with respect to $\left(U_{\alpha}\right)$. An essential step is to show that under $(0.1)$ and $(0.2)$ all points in $E$ are nonbranch points for the associated Ray resolvent (in the usual sense). What remains to be proved is that the corresponding Ray process may be restricted to the state space $(E, \mathfrak{E})$ to yield a right process with resolvent $\left(U_{\alpha}\right)$.

The paper is organized as follows: In the first two sections notation is fixed and some preliminary and auxiliary lemmas are proved. $\S 2$ is of interest mainly in connection with part of $\S 6$, where a generalization of the results on nonbranch points without the assumption that 1 is excessive is discussed. $\S 3$ contains the investigation of nonbranch points, in particular Definition (3.1) and the main characterizations in Theorems (3.3) and (3.5) and in Corollaries (3.4) and (3.6); these are of interest in their own right as well. In $\S 4$ the first result mentioned in (0.7) on the existence of a right process is established, the main theorem being (4.1). The reader who is mainly interested in this result may skip $\S 2$ and restrict the reading of $\S 3$ to the proof of $(a) \Rightarrow(b 1)$ in Theorem (3.3) and the second part of the proof of Theorem (3.5). In $\S 5$ the second result mentioned in (0.8) is proved characterizing the existence of a right continuous semigroup; here the main theorem is $(5.7)$. $\S 6$ is devoted to generalizations, particularly the case where the assumption that the potential kernel is proper is dropped. Here, of course, necessary and sufficient conditions have to be expressed in terms of the $r$-excessive measures for $r>0$. The main results are also stated in this context. Furthermore, concerning the results on nonbranch points, weakening of the hypotheses " $(E, \mathfrak{E})$ Lusin" and " 1 excessive" is discussed (using the preparatory remarks of $\S 2$ ). The paper ends with a few remarks on the definitions of nonbranch points by Engelbert and Wittmann and on their relationship with definition (0.6). 
I thank K. Janssen and R. K. Getoor for helpful remarks and P. J. Fitzsimmons for providing me with another proof of part of Theorem (3.3).

\section{NOTATION AND PRELIMINARIES}

In this section we fix notation, and list some properties of excessive measures and functions that play a role in the sequel. For a general reference concerning resolvents, excessive functions and measures we refer to [4]; in connection with processes we refer as well to $[2,11,7,12,10]$, and concerning especially Ray resolvents and Ray compactifications to [11, 7, 21] in addition to [4].

In the following let $\left(U_{\alpha}\right)_{\alpha>0}$ denote a substochastic resolvent on a measurable space $(E, \mathfrak{E})$ with potential kernel $U$. Our notation for the most part is standard. For example, by $\underline{\underline{E}}, p \underline{\underline{E}}, b \underline{\underline{E}}$ the measurable, resp. positive or bounded measurable, functions are denoted. We use the symbol $\wedge$ to denote the pointwise infimum of two functions; a class of functions is said to be $\wedge$-stable if it is closed under finite infima. For a class $C$ of functions $\Lambda C$ denotes the set of all finite infima in $C$. Following [7], we call a cone $C \subset p b \underline{E}$ a Riesz cone if it is $\wedge$-stable, if $1 \in C$, and if $\mathfrak{E}=\sigma(C)$. If $\{x\} \in \mathbb{E}$ for all $x \in E$ then a Riesz cone separates the points of $E$. Furthermore, a vector lattice $V \subset b \underline{E}$ is called a Stone space if $V$ contains infima with constants and if $\mathbb{E}=\sigma(V)$. In particular, if $C$ is a Riesz cone then $C-C$ forms a Stone space.

(1.1) Recall that the potential kernel $U$ is said to be proper if there exists a strictly positive function $q \in \underline{E}$ such that $U q<\infty$, and that the resolvent is said to be nondegenerate if all kernels $U_{\alpha}$ are nondegenerate, i.e., $U_{\alpha}(x)>$, for all $\alpha>0$ and all $x \in E$. Of course, $\left(U_{\alpha}\right)$ is nondegenerate if 1 is excessive. Following Engelbert [7], we call the resolvent $\left(U_{\alpha}\right)$ normal if the function 1 is excessive with respect to $\left(U_{\alpha}\right)$ and natural if the union of $\mathfrak{E}$-measurable $r$ excessive functions $(r \geq 0)$ generates $\mathfrak{E}$. In case the potential kernel is proper $\left(U_{\alpha}\right)$ is natural if and only if the $\mathfrak{E}$-measurable excessive functions generate $\mathfrak{E}$. Furthermore, following [7], we call a Riesz cone a Ray cone if each of its functions is $\beta$-supermedian for some $\beta \geq 0$ and if $\left(U_{\alpha}\right)$ maps $C$ into itself.

(1.2) Let us denote the class of $\sigma$-finite positive mesures on $(E, \mathfrak{E})$ by $\mathscr{M}^{+}(E)-$ different from usual notation, but more convenient for our purpose since we shall deal only with $\sigma$-finite measures. Furthermore, as usual, let $\mathscr{M}_{1}^{+}(E)$ denote the probability measures on $(E, E)$. Recall that a measure $m \in \mathscr{M}^{+}(E)$ is called $\beta$-excessive with respect to $\left(U_{\alpha}\right)$ if $\alpha m U_{\beta+\alpha}$ increases to $m$ as $\alpha$ increases to $\infty$. According to XII-37 of [4], if the resolvent is nondegenerate then $m \in \mathscr{M}^{+}(E)$ is $\beta$-excessive if and only if it is $\beta$-supermedian, i.e., $\alpha m U_{\beta+\alpha} \leq m$ for all $\alpha>0$.

(1.3) By $\mathscr{S}^{\beta}$ or $\mathscr{S}^{\beta}(U)$ (resp. $\mathscr{E}^{\beta}$ or $\mathscr{E}^{\beta}(U)$ ) we denote the E-measurable $\beta$-supermedian (resp. $\beta$-excessive) functions, and by $\operatorname{Exc}^{\beta}$ or $\operatorname{Exc}^{\beta}(U)$ the $\beta$ excessive measures with respect to $\left(U_{\alpha}\right)$. As usual, when $\beta=0$ we drop the $\beta$ from our notation and terminology. Following [10], we abbreviate the 
subclasses of Exc consisting of potentials and of purely excessive measures by Pot and Pur, respectively. If $f \in \mathscr{S}^{\beta}$ then $\hat{f}=\uparrow \lim _{\alpha} \alpha U_{\alpha+\beta} f$ denotes the $\beta$-excessive regularization of $f$. The symbol $\prec$ is used for the specific order on $\operatorname{Exc}^{\beta}$ (as well as on $\mathscr{E}^{\beta}$ ); i.e., if $\mu, \nu \in \operatorname{Exc}^{\beta}$ then $\mu \prec \nu$ if there exists a $\rho \in \operatorname{Exc}^{\beta}$ such that $\mu+\rho=\nu$ (accordingly for $\mathscr{E}^{\beta}$ ), whereas $\leq$ is used for the simple or natural order on measures (resp. pointwise order on functions).

(1.4) On the cartesian product Exc $\times \mathscr{S}$ of excessive measures and supermedian functions, the energy functional $L$ is defined by (cf. XII-39/40 of [4]):

$$
L(m, f):=L(m, \hat{f}):=\sup \{\mu(\hat{f}): \mu U \in \text { Pot }, \mu U \leq m\} .
$$

It is positive, linear, and increasing in both arguments. In particular, if $m$ or $f$ is a potential, i.e., $m=\mu U \in$ Pot or $f=U g$, then-provided $U$ is proper-the energy functional represents their mass: $L(\mu U, f)=\mu(f)$ and $L(m, U g)=m(g)$. We shall make use of the energy functional only in cases where the potential kernel $U$ is proper; then, according to XII-Theorem 38 of [4], any excessive measure is the increasing limit of potential measuresanalogously as for functions.

(1.5) For easy reference let us fix some abbreviations for properties of $\left(U_{\alpha}\right)$ excessive measures and functions that are of special interest in the following sections:

(IE) $\wedge$-stability of excessive functions: if $f, g \in \mathscr{E}$ then $f \wedge g \in \mathscr{E}$.

(UC) Unicity of charges: if $\mu U=\nu U \in$ Pot then $\mu=\nu$.

(UPC) Unicity of point charges: for any $x \in E$, if $\mu U=\varepsilon_{x} U \in$ Pot then $\mu=\varepsilon_{x}$.

(EPC) Extremality of point charges: for any $x \in E, \varepsilon_{x} U$ lies on an extreme ray of Exc (i.e., whenever $m \in$ Exc with $m \prec \varepsilon_{x} U$ then $m=c \cdot \varepsilon_{x} U$ for some constant $c \in[0,1])$.

(NSP) Natural solidity of potentials: if $m \in$ Exc such that $m \leq \pi \in$ Pot then $m \in$ Pot .

(SSP) Specific solidity of potentials: if $m \in$ Exc such that $m \prec \pi \in$ Pot then $m \in$ Pot .

In addition, for some fixed $x \in E$, we define corresponding "local" properties.

$\left(\mathrm{IE}_{x}\right)$ If $f, g \in \mathscr{E}$ then $\widehat{f \wedge} g(x)=f \wedge g(x)$.

$\left(\right.$ IS $\left._{x}\right)$ The cone $\{f \in b \mathscr{S}: \hat{f}(x)=f(x)\}$ is $\wedge$-stable.

$\left(\mathrm{UPC}_{x}\right.$ ) If $\mu U=\varepsilon_{x} U \in$ Pot then $\mu=\varepsilon_{x}$.

$\left(\mathrm{EPC}_{x}\right) \varepsilon_{x} U$ lies on an extreme ray of Exc.

$\left(\mathrm{NSP}_{x}\right)$ If $m \in$ Exc such that $m \leq \varepsilon_{x} U \in$ Pot then $m \in$ Pot.

$\left(\operatorname{SSP}_{x}\right)$ If $m \in$ Exc such that $m \prec \varepsilon_{x} U \in$ Pot then $m \in$ Pot.

(1.6) Remarks. (1) If $U$ is proper then the property (UC) is equivalent with the property that $\mathscr{E}$ separates the class $\left\{\mu \in \mathscr{M}^{+}(E): \mu U \in \mathscr{M}^{+}(E)\right\}$ of $\sigma$-finite measures that generate potentials. This class then contains all finite measures 
on $E$ : since $U$ is proper there exists a function $g \in p \underline{\underline{E}}$, with $0<g$ and $U g \leq 1$; using the complete maximum principle (cf. XII-Theorem 28 of [4]) one may choose $g:=\sum_{n \geq 1}\left(n \cdot 2^{n}\right)^{-1} \cdot q 1_{\{U q \leq n\}}$ provided $q \in p \underline{\underline{\mathfrak{E}}}$ such that $0<q<\infty$ and $U q<\infty$.

(2) It is immediate that $\left(\mathrm{EPC}_{x}\right)$ implies $\left(\mathrm{SSP}_{x}\right)$ and that $\left(\mathrm{SSP}_{x}\right)$ together with $\left(\mathrm{UPC}_{x}\right)$ implies $\left(\mathrm{EPC}_{x}\right)$. Thus under $\left(\mathrm{UPC}_{x}\right),\left(\mathrm{SSP}_{x}\right)$ and $\left(\mathrm{EPC}_{x}\right)$ are equivalent.

(3) The resolvent of a right process satisfies (IE); furthermore it satisfies (NSP) and (UC) according to the results of [12] mentioned in the introduction. Therefore by (2), since (NSP) trivially implies (SSP), in particular the extremality of point charges (EPC) holds for right processes.

(4) For Ray resolvents, the extremality of point charges (EPC) is violated exactly at "proper" branch points, i.e., where the branch distribution is not just a multiple of a point charge.

(5) Of course, (EPC) does not imply (NSP); see example (1.7.1).

(6) (UC) does not imply (IE), as the example of $\left(U_{\alpha}\right)$ on $E$ in (1.7.3) shows. On the other hand, (IE) does not imply (UC), as the example (1.7.2) of the Ray resolvent on $K$ shows. See, however, Lemma (2.4).

For easy reference we list some examples that may serve to illuminate the relations among the different properties of excessive measures.

(1.7) Examples. (1) Consider the resolvent of translation on $\mathbf{R}$, and restrict it to $\mathbf{R} \backslash 0$. Then there does not exist a right process nor a right semigroup corresponding to this resolvent.

(2) Consider the Ray resolvent of translation to the right on $K:=[0, a] \cup$ $[b, c](0<a<b<c)$, with a branch distribution at $a$ that is (i) unit mass at $b$ or (ii) a proper fraction of unit mass at $b$. Let $\left(U_{\alpha}\right)$ denote the Ray resolvent restricted to the space $E:=K \backslash\{b, c\}$. Then in example (i) $\left(U_{\alpha}\right)$ corresponds to the right process of translation on $E$ with $a$ and $b$ identified; in example (ii), however, $\left(U_{\alpha}\right)$ does not correspond to a right process on $E$, but to a right continuous semigroup (see Definition (5.1)). Here the $\left(U_{\alpha}\right)$-excessive functions are $\wedge$-stable, as well as the excessive functions with respect to the original Ray resolvent on $K$.

(3) Consider the Ray resolvent of translation to the right on the "fork" $K:=$ $[0, a] \cup\left[b_{1}, c_{1}\right] \cup\left[b_{2}, c_{2}\right]$ with a branch distribution at $a$ that is $1 / 2$ of unit mass at both $b_{1}$ and $b_{2}$.

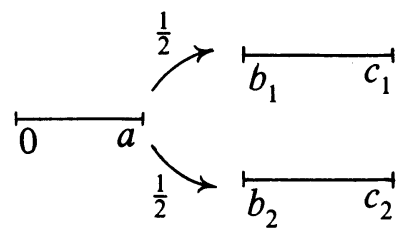

Let $\left(U_{\alpha}\right)$ denote the Ray resolvent restricted to the space

$$
E:=K \backslash\left\{b_{1}, c_{1}, b_{2}, c_{2}\right\} .
$$


Then, as in example $(2),\left(U_{\alpha}\right)$ does not correspond to a right process on $E$, but to a right continuous semigroup. Here the $\left(U_{\alpha}\right)$-excessive functions are not $\wedge$-stable; this is seen by the same example as that usually given for the Ray resolvent on $K: f \equiv 1$ on $[0, a], f \equiv 2$ on $] b_{1}, c_{1}[, f \equiv 0$ on $] b_{2}, c_{2}[$, and $g$ defined the same with only the subscripts at $b$ and $c$ exchanged. Then $f, g \in \mathscr{E}(U)$, but $f \wedge g \notin \mathscr{E}(U)$.

\section{A TRANSFORMATION OF THE RESOLVENT}

In this section we discuss a transformation of the given resolvent into a resolvent with bounded potential kernel and where moreover the function 1 is itself a potential. Getoor and Glover [13], and earlier Meyer [18], made use of this transformation in the situation where the resolvent corresponds to a right process, but one does not need the process for its definition.

(2.0) Let $\left(U_{\alpha}\right)_{\alpha>0}$ denote a substochastic resolvent on a measurable space $(E, \mathfrak{E})$ with proper and nondegenerate potential kernel $U$, and let $q \in p \underline{\underline{E}}$ be such that $q>0$ and $0<U q<\infty$.

From the following lemma it is seen that concerning any of the properties of excessive measures and functions listed in (1.5), one can always restrict one's considerations to the case where the potential kernel maps the function 1 into itself.

(2.1) Lemma. Given assumptions (2.0) there exists a substochastic resolvent $\left(V_{\alpha}\right)_{\alpha>0}$ on $(E, \mathfrak{E})$ with bounded potential kernel $V$ satisfying.

(i) $V f=(U q)^{-1} U(q f)$ for $f \in p \underline{\underline{E}}$, in particular $V 1=1$;

(ii) $\operatorname{Exc}(V)=q \cdot \operatorname{Exc}(U)$;

(iii) $\mathscr{E}(V)=(U q)^{-1} \cdot \mathscr{E}(U), \mathscr{S}(V)=(U q)^{-1} \cdot \mathscr{S}(U)$;

(iv) for $f \in \mathscr{S}(V): \hat{f}=(U q)^{-1} \widehat{U q \cdot f}$, where the first regularization is taken with respect to $\left(V_{\alpha}\right)$, the second with respect to $\left(U_{\alpha}\right)$.

Proof. $V f:=(U q)^{-1} U(q f)$ (for $f \in p \underline{\underline{E}}$ ) defines a positive kernel on $(E, \mathfrak{E}$ ) satisfying $V 1=1$. It is easily seen that $V$ satisfies the complete maximum principle since $U$ satisfies the domination principle, so there exists a substochastic resolvent $\left(V_{\alpha}\right)_{\alpha>0}$ with potential kernel $V$ (cf. XII-28, -88 of [4]). This is (i). The transformation of the corresponding excessive measures in (ii) and of the excessive functions in (iii) follows immediately by approximation by potentials. (Recall that both excessive measures and excessive functions w.r.t. both resolvents $\left(U_{\alpha}\right)$ and $\left(V_{\alpha}\right)$ are increasing limits of potentials since both $U$ and $V$ are proper.) Furthermore, recalling that the regularization of a supermedian function $f$ is the largest excessive function dominated by $f$, one obtains statement (iv). It remains to establish the second equality in (iii). This follows at once from the fact that, roughly speaking, supermedian functions differ from excessive functions only on sets of potential zero, and the latter are the same 
for $U$ and $V$. Precisely:

$$
\begin{aligned}
& \mathscr{S}(V)=\left\{f \in p \underline{\underline{E}}: \text { there exists } g \in \mathscr{E}(V), \quad g \leq f \text { and } V 1_{\{g<f\}}=0\right\} \\
&=\left\{f \in p \mathfrak{E}: \text { there exists } h \in \mathscr{E}(U), h \leq U q \cdot f \text { and } V 1_{\{h<U q \cdot f\}}=0\right\} \\
&=(U q)^{-1} \cdot\{f \in p \underline{\underline{E}}: \text { there exists } h \in \mathscr{E}(U): h \leq f \\
&=(U q)^{-1} \cdot \mathscr{S}(U), \text { because }(U q)^{-1} U\left(q \cdot 1_{\{h<f\}}\right)=0 \\
& \text { if and only if } U\left(1_{\{h<f\}}\right)=0 .
\end{aligned}
$$

In the sequel, without special mention, $\left(V_{\alpha}\right)$ will always denote the resolvent constructed from $\left(U_{\alpha}\right)$ as just explained.

(2.2) Remarks. (1) As is immediate from (2.1), the transformation relating $\left(V_{\alpha}\right)$ to $\left(U_{\alpha}\right)$ leaves invariant each of the properties of excessive measures and functions listed in (1.5).

(2) A property, however, that is not invariant under that transformation is that the excessive functions generate the $\sigma$-algebra $\mathfrak{E}$ (i.e., that the resolvent is natural). Consider for an example the Ray resolvent of translation on $K$ as described in (1.7.2(ii)).

(3) Another property that is not invariant under that transformation is that the vector space of differences of bounded excessive functions forms an algebra; this can be seen from the same example (1.7.2(ii)) as before.

(2.3) Denote by $\mathfrak{E}^{\prime}$ the $\sigma$-algebra generated by the class $\{f / g: f, g \in \mathscr{E}(U)$, $g>0\}$ of quotients in $\mathscr{E}(U)$.

Obviously, $\mathfrak{E}^{\prime}$ coincides with the $\sigma$-algebra generated by $\mathscr{E}(V)$. The hypothesis $\mathfrak{E}=\mathfrak{E}^{\prime}$ then means that $\left(V_{\alpha}\right)$ is natural (see 1.1). Furthermore, $\mathfrak{E}=\mathfrak{E}^{\prime}$ implies of course that $\left(U_{\alpha}\right)$ is natural as well, since $\mathfrak{E} \supset \sigma(\mathscr{E}(U)) \supset \sigma(\mathscr{E}(V))=\mathfrak{E}^{\prime}$. On the other hand, $\left(U_{\alpha}\right)$ natural does not imply $\mathfrak{E}=\mathfrak{E}^{\prime}$, as the example mentioned above shows. This is the case, however, if $\left(U_{\alpha}\right)$ is moreover normal, i.e., if $1 \in \mathscr{E}(U)$, but this latter condition is not necessary for $\mathfrak{E}=\mathfrak{E}^{\prime}$ (consider the resolvent $\left(U_{\alpha}\right)$ on $E$ in example (1.7.2(ii)) again). See also Lemma (2.5).

In the following sections we are going to work under the hypotheses $\left(U_{\alpha}\right)$ natural and normal (i.e., $\mathfrak{E}=\sigma(\mathscr{E}(U)$ ) and $1 \in \mathscr{E}(U)$ ). For most of the results on nonbranch points in $\S \S 3$ and 5 , these could be replaced by the weaker hypothesis $\left(V_{\alpha}\right)$ natural (see $\S 6$ for a discussion); but for the main result in $\S 4$ characterizing the existence of a right process the assumption $1 \in \mathscr{E}(U)$ is necessary.

Recall the definitions of properties (IE), (UC), (UPC) from (1.5).

(2.4) Lemma. In addition to hypotheses (2.0) assume $\mathfrak{E}=\mathfrak{E}^{\prime}$. Then (IE) implies (UC).

Proof. According to (2.2) and the related remarks it suffices to prove the statement for the resolvent $\left(V_{\alpha}\right)$. Let $\mu V=\nu V \in \operatorname{Pot}(V)$, and consider the class 
$\tilde{\mathscr{E}}:=\{f \in \mathscr{E}(V): f$ bounded and $\mu(f)<\infty\}$. Then under the hypotheses in the statement, (IE) implies that $\tilde{\mathscr{E}}-\tilde{\mathscr{E}}$ forms a Stone space. $(\widetilde{\mathfrak{E}}=\sigma(\widetilde{\mathscr{E}})$ follows because any $f \in \mathscr{E}(V)$ is the increasing limit of a sequence in $\tilde{\mathscr{E}}$.) Since $\mu$ agrees with $\nu$ on $\widetilde{\mathscr{E}}-\tilde{\mathscr{E}}, \mu=\nu$ on $\mathfrak{E}$ follows by uniqueness in the Daniell-Stone extension theorem (see, e.g., [1]).

(2.5) Lemma. In addition to hypotheses (2.0) assume that $(E, \mathfrak{E})$ is a Souslin space, $\left(U_{\alpha}\right)$ is natural, and (UPC) holds for $\left(U_{\alpha}\right)$. Then $\mathfrak{E}=\mathfrak{E}^{\prime}$.

Proof. First note that under (UPC), $\mathscr{E}(V)$ separates $\mathscr{E}$. This follows because, if $x, y \in E$ were not separated by $\mathscr{E}(V)$, then $\varepsilon_{x} V=\varepsilon_{y} V$ and by (UPC) (which holds for $\left(V_{\alpha}\right)$ whenever it holds for $\left(U_{\alpha}\right)$ ) one obtains $\varepsilon_{x}=\varepsilon_{y}$ on $\mathfrak{E}$, i.e., $x=y$ since $\mathfrak{E}$ separates points. Now both measurable spaces $(E, \mathfrak{E})$ and $\left(E, \mathfrak{E}^{\prime}\right)$ are separable and separated and $(E, \mathfrak{E})$ is Souslin. Therefore the Souslin-Lusin Theorem (cf. III.21 of [3]) applied to the identity map from $(E, \mathfrak{E})$ onto $\left(E, \mathfrak{E}^{\prime}\right)$ yields $\mathfrak{E}=\mathfrak{E}^{\prime}$.

\section{NONBRANCH POINTS: CHARACTERIZATION BY EXCESSIVE MEASURES}

Throughout this and the following sections we consider the situation:

(3.0) $\left(U_{\alpha}\right)_{\alpha>0}$ is a natural and normal (see 1.1) substochastic resolvent on a Lusin space $(E, \mathfrak{E})$ with a proper potential kernel $U$.

Here Lusin means measure isomorphic to a Borel set in some compact metric space (cf. III-16b) of [3]).

We are going to define a notion of a nonbranch point for $\left(U_{\alpha}\right)$ in terms of the $\left(U_{\alpha}\right)$-excessive measures. Under hypotheses (3.0) this definition seems to be appropriate, and moreover it turns out to coincide with previous definitions given by Engelbert [7] and Wittmann [21]. See $\S 6$ for a discussion.

(3.1) Definition. We call a point $x \in E$ a nonbranch point for $\left(U_{\alpha}\right)$ if property $\left(\mathrm{EPC}_{x}\right)$ holds, i.e., if $\varepsilon_{x} U$ lies on an extreme ray of $\operatorname{Exc}(U)$ (see 1.5).

Under (3.0), according to Engelbert (see the proof of Theorem 9 in VI of [7]), the collection $C\left(U_{\alpha}(b \mathfrak{E})\right):=\left\{c \wedge f: c \in \mathbf{R}_{+}\right.$and $\left.f \in \Lambda\left(U_{\alpha}(b \mathfrak{E})\right), f \geq 0\right\}$ forms a Ray cone for $\left(U_{\alpha}\right)$, which contains a separable Ray cone. Fix such a separable Ray cone $C$ in $C\left(U_{\alpha}(b \mathfrak{E})\right)$. Then according to VI- $\S 9$ of [7], analogously as in [11], there exists a Ray compactification $(K, \mathfrak{B}(K))$ of $(E, \mathfrak{E})$ with respect to $C$ with Ray resolvent $\left(\bar{U}_{\alpha}\right)$ extending $\left(U_{\alpha}\right)$. Here $\mathfrak{B}(K)$ is the Borel $\sigma$-algebra in $K$ with respect to the Ray topology on $K$ induced by $C$. Let us denote by $\left(\bar{P}_{t}\right)_{t \geq 0}$ the associated Ray semigroup and by $D$ the set of "nonbranch points" with respect to $\left(\bar{U}_{\alpha}\right)$ as usually defined in the Ray context; i.e., $x \in D$ if $\lim _{\alpha} \alpha \bar{U}_{\alpha}(x, f)=f(x)$ for any Ray continuous $f$ on $K$ or, equivalently, if $\varepsilon_{x} \bar{P}_{0}=\varepsilon_{x}$. Let $\bar{C}$ denote the cone of Ray continuous extensions $\bar{f}$ to $K$ of functions $f \in C$ on $E$.

(3.2) Remarks (see Chapter VI of [7]). (1) Since $C$ is separable $K$ is metrizable and $\mathfrak{B}(K)=\sigma(\bar{C}) . \bar{C}$ is a Ray cone with respect to $\left(\bar{U}_{\alpha}\right)$; in particular, $\bar{C}$ 
separates $K$ and $\bar{C}-\bar{C}$ is dense in the class of Ray continuous functions on $K$.

(2) For any $f \in C, \bar{U}_{\alpha} \bar{f}:=\overline{U_{\alpha} f}$. For any $x \in E, \varepsilon_{x} \bar{U}=\varepsilon_{x} U . D \in \mathfrak{B}(K)$, and $\left(\bar{U}_{\alpha}\right)$ and $\left(\bar{P}_{t}\right)$ are concentrated on $D$; i.e., for any $x \in K, \bar{U}_{\alpha}(x, K \backslash D)=$ $0=\bar{P}_{t}(x, K \backslash D)$ for all $\alpha$ and $t$. Moreover, restricted to $D,\left(\bar{P}_{t}\right)$ is the semigroup of a right process on $D$ with resolvent $\left(\bar{U}_{\alpha}\right)$.

(3) $\mathfrak{B}(K) \cap E=\mathfrak{E}$; moreover-since $(E, \mathfrak{E})$ is Lusin- $E \in \mathfrak{B}(K)$.

(4) We may and will assume in the following that there do not exist degenerate branch points for $\left(\bar{U}_{\alpha}\right)$ (i.e., points $x \in K$ such that $\varepsilon_{x} \bar{P}_{0}=\varepsilon_{y}$ and $y \neq x$ ). This can always be achieved by passing over to the quotient space $K / \sim$ with respect to the equivalence relation: $x \sim y$ if $\varepsilon_{x} \bar{U}_{\alpha}=\varepsilon_{y} \bar{U}_{\alpha}$ for some (all) $\alpha>0$. Since under (3.0), $\mathscr{E}(U)$ separates the points of $E$, this equivalence relation distinguishes the points of $E$.

The subsequent result gives a characterization of nonbranch points for $\left(U_{\alpha}\right)$. Statement (b2) being equivalent with the others was shown to me by P. J. Fitzsimmons, who furthermore kindly allowed me to publish his proof of (a) $\Rightarrow$ (b2) $\Rightarrow(\mathrm{c})$.

(3.3) Theorem. Under the hypotheses (3.0), for $x \in E$ the following statements are equivalent:

(a) $x$ is a nonbranch point for $\left(U_{\alpha}\right)$;

(b1) $x \in D$, i.e., $\varepsilon_{x} \bar{P}_{0}=\varepsilon_{x}$;

(b2) $\widehat{f \cdot g}(x)=f \cdot g(x)$ for any $f, g \in \mathscr{E}(U)$;

(c) $\widehat{f \wedge} g(x)=f \wedge g(x)$ for any $f, g \in \mathscr{E}(U)$;

(d) for any $f \in \Lambda \mathscr{E}(U), \hat{f}(x)=f(x)$;

(e) there exists a $\wedge$-stable cone $C_{0} \subset\{f \in b \mathscr{S}(U): \hat{f}(x)=f(x)\}$ such that $b \mathscr{E}(U) \subset C_{0}$;

(f) the cone $C_{x}:=\{f \in b \mathscr{S}(U): \hat{f}(x)=f(x)\}$ is $\wedge$-stable.

Remark. This means, in particular, that under (3.0) the properties $\left(\mathrm{EPC}_{x}\right)$, $\left(\mathrm{IE}_{x}\right)$, and $\left(\mathrm{IS}_{x}\right)$ from (1.5) are equivalent among each other and, moreover, are equivalent with a localized version (b2) of $b \mathscr{E}(U)-b \mathscr{E}(U)$ being an algebra. Note that the equivalence of (b1) and (b2) with the other statements relies on the fact that the function 1 is excessive, i.e., $\left(U_{\alpha}\right)$ normal, and that (b1) and (b2) are not invariant under the transformation of $\left(U_{\alpha}\right)$ to $\left(V_{\alpha}\right)$ in (2.1). (Consider, e.g., the resolvent $\left(U_{\alpha}\right)$ on $E$ in example (1.7.2(ii)).) See (6.7), (6.9) for generalizations of (3.3).

Proof. We are going to prove $(\mathrm{a}) \Rightarrow(\mathrm{b} 2) \Rightarrow(\mathrm{c})$ first, then $(\mathrm{a}) \Rightarrow(\mathrm{b} 1) \Rightarrow(\mathrm{c})$, and finally the equivalence of $(c)-(f)$ with (a).

(a) $\Rightarrow(\mathrm{b} 2) \Rightarrow(\mathrm{c})$ (Fitzsimmons): First of all note that $b \mathscr{S}-b \mathscr{S}$ is a vector lattice and moreover an algebra. The latter follows from the fact that for any $f \in-b \mathscr{S}$ by Jensen's inequality $f^{2} \in-b \mathscr{S}$ follows. Extending the regularization - to $b \mathscr{S}-b \mathscr{S}$ by linearity yields $\hat{g}(x)=\lim _{\alpha} \alpha U_{\alpha} g(x)$ for any 
$g \in b \mathscr{S}-b \mathscr{S}$. This shows that ${ }^{-}$is monotone on $b \mathscr{S}-b \mathscr{S}$ and that the mapping $g \mapsto \hat{g}(x)$ is continuous in the uniform norm on $b \mathscr{S}-b \mathscr{S}$. Now fix $g \in b \mathscr{S}-b \mathscr{S}$ with $0 \leq g \leq 1$, and define $m_{1}(f):=\widehat{g \cdot U f}(x)$, $m_{2}(f):=\overline{(1-g) \cdot U f}(x)$ for $f \in p \underline{\underline{E}}$. Clearly, each $m_{i}$ is additive and $m_{1}+m_{2}=\varepsilon_{x} U$; thus each $m_{i}$ defines a measure on $(E, \mathfrak{E})$, which is moreover excessive, obviously. By (a), i.e., by $\left(\mathrm{EPC}_{x}\right), m_{i}=c_{i} \varepsilon_{x} U$ for $c_{i} \in[0,1]$, and here $c_{1}+c_{2}=1$ since $1 \in \mathscr{E}(U)$.

Choosing a sequence $\left(U f_{n}\right)$ of potentials increasing to 1 , we obtain $c_{1} U f_{n}(x)$ $=m_{1}\left(f_{n}\right)=\widehat{g \cdot U f_{n}}(x) \leq \hat{g}(x)$, and thus $c_{1} \leq \hat{g}(x) ;$ analogously, $c_{2} \leq$ $(\widehat{1-g})(x)=1-\hat{g}(x)$. This implies $c_{1}=\hat{g}(x)$ and $c_{2}=1-\hat{g}(x)$. Hence we obtain

$$
\widehat{g \cdot U f}(x)=\hat{g}(x) \cdot U f(x) \text { for all } g \in b \mathscr{S}-b \mathscr{S} \text { and all } f \in p \underline{\underline{E}} \text {. }
$$

Let $f \in \mathscr{E}$ be the increasing limit of potentials $U f_{n}$; then, as above,

$$
\widehat{g \cdot f}(x)=\widehat{\lim _{n} g \cdot U f_{n}}(x) \leq \lim _{n} \widehat{g \cdot U f_{n}}(x)=\lim _{n} \hat{g}(x) \cdot U f_{n}(x)=\hat{g}(x) \cdot f(x)
$$

and analogously $\overline{(-g) \cdot f}(x) \leq(1-\hat{g}(x)) \cdot f(x)$, which implies:

$(* *) \quad \widehat{g \cdot f}(x)=\hat{g}(x) \cdot f(x)$ for all $g \in b \mathscr{S}-b \mathscr{S}$ and all $f \in b \mathscr{E}-b \mathscr{E}$

(after taking differences). This, in particular, yields (b2). Since, on the other hand, for $f, g \in b \mathscr{S}, \widehat{f \cdot g}(x)=\widehat{\hat{f} \cdot \hat{g}}(x),(\mathrm{b} 2)$ implies $\widehat{f \cdot g}(x)=\hat{f}(x) \cdot \hat{g}(x)$ for all $f, g \in b \mathscr{S}-b \mathscr{S}$ and, in particular, is equivalent with (**). Hence, from (b2) by induction on (**) we obtain $\widehat{f}^{n}(x)=(f(x))^{n}$ for all $f \in b \mathscr{E}$ $b \mathscr{E}, n \in \mathbf{N}$. Therefore $\widehat{p \circ f}(x)=p \circ f(x)$ for all polynomials $p$ and all $f \in b \mathscr{E}-b \mathscr{E}$. Now, given $f \in b \mathscr{E}-b \mathscr{E}$, uniform approximation of the absolute value function on $[-\|f\|,\|f\|]$ by a sequence of polynomials $\left(p_{n}\right)$ leads to

$$
\widehat{|f|}(x)=\varlimsup_{n} p_{n} \circ f(x)=\lim _{n} \widehat{p_{n} \circ f}(x)=\lim _{n} p_{n} \circ f(x)=|f|(x) .
$$

Since $f \wedge g=\frac{1}{2}(f+g-|f-g|)$ the desired result (c) follows for any two functions $f, g \in b \mathscr{E}$ and thus for any $f, g \in \mathscr{E}$.

(a) $\Rightarrow($ b 1$) \Rightarrow\left(\right.$ c). Recall the properties of the Ray resolvent $\left(\bar{U}_{\alpha}\right)$ on $(K$, $\mathfrak{B}(K))$. Since $\mathfrak{E}=\mathfrak{B}(K) \cap E$ and $E \in \mathfrak{B}(K)$ we may and will identify measures on $K$ that are carried by $E$ with their restrictions to $E$. We claim that $\varepsilon_{x} \bar{P}_{0}=$ $\varepsilon_{x}$ provided $\left(\mathrm{EPC}_{x}\right)$ holds. Let $A \in \mathfrak{B}(K)$ and define $\mu_{1}:=\varepsilon_{x} \bar{P}_{0 \mid A}$ and $\mu_{2}:=\varepsilon_{x} \bar{P}_{0 \mid A^{c}}$ as measures on $(K, \mathfrak{B}(K))$ carried by $D$. Then we have $\varepsilon_{x} U=$ $\varepsilon_{x} \bar{U}=\varepsilon_{x} \bar{P}_{0} \bar{U}=\left(\mu_{1}+\mu_{2}\right) \bar{U}$. Hence $\mu_{i} \bar{U}$ is concentrated on $E$ for $i=1,2$; as a measure on $E, \mu_{i} \bar{U}$ is furthermore excessive with respect to $\left(U_{\alpha}\right)$ since $\mu_{i} \bar{U} U_{\alpha}=\mu_{i} \overline{U U_{\alpha}}$; besides $\mu_{1} \bar{U}+\mu_{2} \bar{U}=\varepsilon_{x} U$. Consequently, $\left(\mathrm{EPC}_{x}\right)$ implies $\mu_{i} \bar{U}=c_{i} \varepsilon_{x} U$ with constants $c_{i} \in[0,1]$. Hence $c_{2} \mu_{1} \bar{U}=c_{1} \mu_{2} \bar{U}$ as measures on $\mathfrak{B}(K)$ carried by $D$. By the principle of unicity of charges (UC) for $\left(\bar{U}_{\alpha}\right)$ 
on $D$ (see 1.6.3) we must have $c_{2} \mu_{1}=c_{1} \mu_{2}$, and since both measures are carried by disjoint sets both are zero. Since $\varepsilon_{x} \bar{P}_{0} \neq 0$ we conclude that either $c_{1}=0$ and $\varepsilon_{x} \bar{P}_{0}(A)=0$ or $c_{2}=0$ and $\varepsilon_{x} \bar{P}_{0}\left(A^{c}\right)=0$ hold. But $A \in \mathfrak{B}(K)$ was arbitrary, so $\bar{P}_{0}(x)=,\gamma \varepsilon_{y}$ for some $y \in D$ and some $\left.\left.\gamma \in\right] 0,1\right]$, and because $1 \in \mathscr{E}(U)$ we must have $\gamma=1$, thus $y=x$ since there do not exist degenerate branch points for $\left(\bar{U}_{\alpha}\right)$; this is (b1).

Furthermore, since restricted to $D,\left(\bar{U}_{\alpha}\right)$ is the resolvent of a right process any two $\left(\bar{U}_{\alpha}\right)$-excessive functions $h, k$ satisfy $h \wedge k(x)=\widehat{h \wedge k}(x)$ for all $x \in D$. Now let $f, g \in \mathscr{E}(U)$ be the increasing limits of potentials $U f_{n}$, resp. $U g_{n}$, and extend $f_{n}$ and $g_{n}$ to $K$ by setting them equal to zero on $K \backslash E$. Denoting the extensions by $\bar{f}_{n}, \bar{g}_{n}$ we obtain $\left(\bar{U}_{\alpha}\right)$-excessive functions $\bar{f}:=\uparrow \lim _{n} \bar{U} \bar{f}_{n}$ and $\bar{g}:=\uparrow \lim _{n} \bar{U}_{\bar{g}}$ which on $E$ agree with $f$ and $g$, respectively. Hence since $x \in E \cap D$ one obtains

$$
\begin{aligned}
\widehat{f \wedge} g(x) & =\lim _{\alpha} \alpha U_{\alpha}(f \wedge g)(x)=\lim _{\alpha} \alpha \bar{U}_{\alpha}(\bar{f} \wedge \bar{g})(x) \\
& =\widehat{f \wedge} \bar{g}(x)=\bar{f} \wedge \bar{g}(x)=f \wedge g(x) .
\end{aligned}
$$

(c) $\Rightarrow$ (d) by induction.

(d) $\Rightarrow$ (e) by defining $C_{0}:=\Lambda b \mathscr{E}(U)$.

(e) $\Rightarrow(\mathrm{f})$ : Let $f, g \in b \mathscr{S}(U)$ satisfying $\hat{f}(x)=f(x)$ and $\hat{g}(x)=g(x)$. Then $\hat{f}, \hat{g} \in b \mathscr{E}(U)$, and $\Lambda b \mathscr{E}(U) \subset C_{0}$. Hence $f \wedge g(x)=\hat{f} \wedge \hat{g}(x)=$ $\widehat{f \wedge} \hat{g}(x) \leq \widehat{f \wedge} g(x) \leq f \wedge g(x)$.

(f) $\Rightarrow(\mathrm{a})$ : Since $1 \in \mathscr{E}(U)$ and $\mathscr{E}(U)$ generates $\mathfrak{E}$, the cone $C_{x}$ in (f) is a Riesz cone. Let $m_{1}, m_{2} \in \operatorname{Exc}(U)$ and $\varepsilon_{x} U=m_{1}+m_{2}$. Then, with the energy functional $L$ on $\operatorname{Exc} \times \mathscr{S}$ (see 1.4) we have for any $f \in C_{x}: f(x)=$ $\hat{f}(x)=L\left(\varepsilon_{x} U, \hat{f}\right)=L\left(\varepsilon_{x} U, f\right)=L\left(m_{1}, f\right)+L\left(m_{2}, f\right)$. Now $L\left(m_{i}, \cdot\right)$ form positive linear functionals on the Stone space $C_{x}-C_{x}$ the sum of which is $\varepsilon_{x}$. Consequently, by the Daniell-Stone extension theorem (see, e.g., [1]) each is represented by a measure $\nu_{i}$ on $\mathfrak{E}$. Hence on $\mathfrak{E}, \varepsilon_{x}=\nu_{1}+\nu_{2}$, implying $\nu_{i}=c_{i} \varepsilon_{x}$ for $i=1,2$ and $c_{i} \in[0,1]$ with $c_{1}+c_{2}=1$. But then $m_{i}=\nu_{i} U=$ $c_{i} \varepsilon_{x} U$, which completes the proof.

In the following let us denote by $N$ the set of nonbranch points for $\left(U_{\alpha}\right)$. Then, by the equivalence of (a) and (d) in Theorem (3.3) it is seen that $N$ can be represented as the intersection over all $f \in \Lambda \mathscr{E}(U)$ of the sets $\{\hat{f}=f\}$. Since $\mathfrak{E}$ is countably generated we may as well choose a countable subcone $\left\{f_{n}: n \in \mathbf{N}\right\}$ of $\Lambda b \mathscr{E}(U)$ to represent $N$, i.e., $N=\bigcap_{n}\left\{\hat{f}_{n}=f_{n}\right\}$. Define $g:=\sum_{n} 2^{-n}\left\|f_{n}\right\|^{-1} \cdot f_{n} ;$ then $\{\hat{g} \neq g\}=\bigcup_{n}\left\{\hat{f}_{n} \neq f_{n}\right\}$ and hence $N=\{\hat{g}=g\}$ as well for one single function $g \in b \mathscr{S}(U)$ that is the increasing limit of the functions $\sum_{k=1}^{n} 2^{-k}\left\|f_{k}\right\|^{-1} \cdot f_{k}$. This shows particularly that $N$ is measurable in $(E, \mathcal{E})$. Since for any $f \in \mathscr{S}(U), U_{\alpha} \hat{f}=U_{\alpha} f$, we conclude that $\left(U_{\alpha}\right)$ has its mass concentrated on $N$, and so does any $\left(U_{\alpha}\right)$-excessive measure. From the equivalence of $(3.3)(\mathrm{a})$ and (c) it is seen by Lemma (2.4) that for $\left(U_{\alpha}\right)$ on $N$ 
the principle of unicity of charges (UC) holds. Summing up these observations we obtain:

(3.4) Corollary. The set $N$ of nonbranch points for $\left(U_{\alpha}\right)$ has the following properties:

(a) There exist a countable subcone $C_{0} \subset \Lambda b \mathscr{E}(U)$ and some function $g \in$ $b \mathscr{S}(U)$ that is the increasing limit of functions in $C_{0}$ such that

$$
N=\bigcap_{f \in \Lambda \mathscr{E}(U)}\{\hat{f}=f\}=\bigcap_{f \in C_{0}}\{\hat{f}=f\}=\{\hat{g}=g\} ;
$$

in particular $N \in \mathbb{E}$.

(b) For any $x \in E, U_{\alpha}(x, E \backslash N)=0$ for all $\alpha \geq 0$, and for any $m \in \operatorname{Exc}(U)$, $m(E \backslash N)=0$.

(c) (UC) holds for $\left(U_{\alpha}\right)$ on $N$; i.e., whenever $\mu, \nu \in \mathscr{M}^{+}(E)$ are concentrated on $N$ with $\mu U=\nu U \in \operatorname{Pot}(U)$, then $\mu=\nu$.

Another characterization of nonbranch points in terms of excessive measures is the following:

(3.5) Theorem. Let $x \in E$. Then under (3.0), $x$ is a nonbranch point for $\left(U_{\alpha}\right)$, i.e., $x \in N$, if and only if properties $\left(\mathrm{UPC}_{x}\right)$ and $\left(\mathrm{SSP}_{x}\right)$ hold. (See 1.5 for definitions.)

Proof. To prove necessity assume $\left(\mathrm{EPC}_{x}\right)$. Then $\left(\mathrm{SSP}_{x}\right)$ follows, and it remains to show $\left(\mathrm{UPC}_{x}\right)$. For this let $\varepsilon_{x} U=\nu U \in$ Pot for some $\nu \in \mathscr{M}^{+}(E)$. According to (3.3.b1), $\varepsilon_{x} \bar{P}_{0}=\varepsilon_{x}$, hence $\nu \bar{P}_{0}$ and $\varepsilon_{x}$ are measures on $D$ such that $\nu \bar{P}_{0} \bar{U}=\varepsilon_{x} \bar{U}$ holds. But (UC) for $\left(\bar{U}_{\alpha}\right)$ restricted to $D$ implies $\nu \bar{P}_{0}=\varepsilon_{x}$. Therefore $\varepsilon_{y} \bar{P}_{0}=\gamma(y) \cdot \varepsilon_{x}$ with $\gamma(y) \in[0,1]$, for $\nu$-almost all $y \in K$. Since $1 \in \mathscr{E}, \nu \in \mathscr{M}_{1}^{+}(E)$, i.e., $\nu$ is a probability measure on $E$; thus $\bar{P}_{0} 1_{K}=1$ implies that for $\nu$-almost all $y \in K$ we have $\gamma(y)=\bar{P}_{0} 1_{K}(y)=1$; however, there are no degenerate branch points, and $\nu=\varepsilon_{x}$ follows. Second, to prove sufficiency assume $\left(\mathrm{UPC}_{x}\right)$ and $\left(\mathrm{SSP}_{x}\right)$ and let $\varepsilon_{x} U=m_{1}+m_{2}$ with $m_{i} \in \operatorname{Exc}$. Then by $\left(\operatorname{SSP}_{x}\right), m_{i}=\nu_{i} U \in$ Pot for $i=1,2$, and furthermore by $\left(\mathrm{UPC}_{x}\right) \varepsilon_{x}=\nu_{1}+\nu_{2}$ follows. Consequently, $\nu_{i}=c_{i} \varepsilon_{x}$ with $c_{i} \in[0,1]$.

As a consequence of Theorems (3.3) and (3.5) the following characterization of the situation where all points in $E$ are nonbranch points is immediate.

(3.6) Corollary. The following properties with respect to the resolvent $\left(U_{\alpha}\right)$ are equivalent ( under (3.0)):

(a) All points in $E$ are nonbranch points;

(b1) $E \subset D$;

(b2) $b \mathscr{E}-b \mathscr{E}$ forms an algebra;

(c) $\mathscr{E}(U)$ is ^-stable;

(d) (SSP) and (UC) hold;

(e) (SSP) and (UPC) hold. 
Proof. The equivalence of the first four statements results from Theorem (3.3); (e) $\Rightarrow$ (a) by (3.5); (d) $\Rightarrow$ (e) is trivial. It remains to show (c) $\Rightarrow$ (d). But under (c), $b \mathscr{E}(U)$ forms a Riesz cone, and an argument analogous to the proof of (f) $\Rightarrow$ (a) in (3.3) gives (SSP); (UC) finally follows according to (2.4).

\section{EXISTENCE OF A BOREL RIGHT PROCESS}

In this section the setup is the same as in $\S 3$.

(4.0) We assume we are given a resolvent $\left(U_{\alpha}\right)$ on a Lusin space $(E, \mathfrak{E})$ subject to hypotheses (3.0).

We are going to give a characterization of when there exists a Borel right process on $(E, \mathfrak{E})$ with resolvent $\left(U_{\alpha}\right)$ in terms of conditions on the $\left(U_{\alpha}\right)$ excessive measures. Recall the definitions of properties (UC), (UPC), (SSP), and (NSP) from (1.5) and definition (3.1) of a nonbranch point for $\left(U_{\alpha}\right)$. As mentioned in Remark (1.6.3), if $\left(U_{\alpha}\right)$ corresponds to a right process on $(E, \mathfrak{E})$ then $\left(U_{\alpha}\right)$ satisfies both (UC) and (NSP), hence also (UPC). Using the same notation as in $\S 3$, by $(K, \mathfrak{B}(K))$ we denote the Ray compactification of $E$ with respect to a fixed Ray cone $C \subset C\left(U_{\alpha}(b \underline{\underline{\mathfrak{E}}})\right)$ (as below 3.1), $\left(\bar{U}_{\alpha}\right)$ and $\left(\bar{P}_{t}\right)$ denote the associated Ray resolvent, resp. semigroup, and $D=\{x \in$ $\left.K: \bar{P}_{0}(x)=,\varepsilon_{x}\right\}$. Recall the properties of these objects as listed in (3.2).

The next theorem establishes the main result that properties (UPC) and (NSP) are not only necessary but also sufficient for the existence of a right process corresponding to the given resolvent.

(4.1) Theorem. Under hypotheses (3.0), there exists a (transient) Borel right process on $(E, \mathfrak{E})$ with resolvent $\left(U_{\alpha}\right)$ if and only if (UPC) and (NSP) are satisfied with respect to $\left(U_{\alpha}\right)$.

Remark. Here "there exists a Borel right process" means: there exists a topology on $E$ that generates the given Borel structure, and there exists a strong Markov process on $(E, E)$ that is a Borel right process (in the sense of [11]) in this topology.

Proof. The necessity is well known from [12]; see Remark (1.6.3). To prove sufficiency observe first that according to (3.6) (NSP) and (UPC) imply $E \subset D$. Now let $Y$ denote the Ray process on $(K, \mathfrak{B}(K))$ associated with $\left(\bar{U}_{\alpha}\right)$ and $X$ its restriction to $D$. Recall that $E \in \mathfrak{B}(K)$ and $E \cap \mathfrak{B}(K)=\mathfrak{E}$ such that the restriction of a measure on $(K, \mathfrak{B}(K))$ to $E$ forms a measure on $(E, \mathfrak{E})$ and vice versa. Again, as in the proof of (3.3), we identify measures on $K$ that are concentrated on $E$ with their restrictions. We claim that for any measure $\mu \in \mathscr{M}_{1}^{+}(E), \mu P_{L}$ is the zero measure for any compact set $L$ in $K \backslash E$ (where $P_{L}$ denotes the hitting kernel for the set $L$ with respect to the process $X)$. Since $\mu P_{L} \bar{U} \leq \mu \bar{U}=\mu U$, which is concentrated on $E, \mu P_{L} \bar{U}$ is concentrated on $E$ as well. Moreover, it is excessive with respect to $\left(U_{\alpha}\right)$ 
because $\mu P_{L} \bar{U} U_{\alpha}=\mu P_{L} \overline{U U}_{\alpha}$, and $\mu P_{L} \bar{U} \leq \mu U$; so by (NSP) $\mu P_{L} \bar{U}$ is a potential, say $\nu U \in \operatorname{Pot}(U)$. Then $\nu \bar{U}$ equals $\mu P_{L} \bar{U}$ since both measures are concentrated on $E$ and agree on $E$; hence applying the unicity of charges (UC) w.r.t. $\left(\bar{U}_{\alpha}\right)$ on $D$ (see Remark 1.6.3) we obtain $\nu=\mu P_{L}$. But both measures are carried by disjoint sets, thus both are zero. Now (by the assumption $(E, \mathfrak{E})$ Lusin) the set $D \backslash E$ is Borel in $K$ as well; therefore according to Theorem 12.15 of [11], there exists an increasing sequence $\left(L_{n}\right)$ of compact sets in $D \backslash E$ such that the hitting times $T_{L_{n}}$ decrease to $T_{D \backslash E}$ a.s. $P^{\mu}$. Hence,

$$
P^{\mu}\left(T_{D \backslash E}<\infty\right)=\uparrow \lim P^{\mu}\left(T_{L_{n}}<\infty\right)=0,
$$

and $\mu P_{D \backslash E}=0$ follows. Consequently, for any $\mu \in \mathscr{M}_{1}^{+}(E)$, a.s. $P^{\mu}$ the process $X$ does not hit the set $D \backslash E$; i.e., $D \backslash E$ is $P^{\mu}$-evanescent for $X$. Therefore the restriction of $X$ to paths in $E$ yields a Borel right process on $(E, \mathfrak{E})$ with resolvent $\left(U_{\alpha}\right)$.

We are going to list some easy consequences of Theorem (4.1).

(4.2) Corollary. Under hypotheses (3.0), $\left(U_{\alpha}\right)$ is the resolvent of a (transient) Borel right process on $(E, \mathfrak{E})$ if and only if the excessive functions are $\wedge$-stable and the measure potentials satisfy the solidity property (NSP).

Proof. This follows immediately from Theorem (4.1), Lemma (2.4), and Remark (1.6.3).

In Chapter II, $\S 3$ of [7] Engelbert introduces the term "standard resolvent"; he shows (see Theorem 9 there) that a resolvent is standard if and only if it is normal and natural (see 1.1) and if $\mathscr{E}^{r}$ is $\wedge$-stable for all $r \geq 0$. In case the potential kernel is proper it is easily seen that $\mathscr{E}^{r} \wedge$-stable for all $r \geq 0$ is equivalent with $\mathscr{E} \wedge$-stable (see also 6.1.3). Hence for standard resolvents the result (4.2) reads as follows.

(4.3) Corollary. $A$ standard resolvent $\left(U_{\alpha}\right)$ with proper potential kernel on a Lusin space $(E, \mathfrak{E})$ is the resolvent of a (transient) Borel right process if and only if the property (NSP) holds for $\left(U_{\alpha}\right)$.

Furthermore, result (4.1) allows us to characterize the subspaces of compact metric spaces that may serve as state spaces of right processes with respect to a given Ray resolvent.

(4.4) Corollary. Let $K$ be a compact metric space, and let $\left(W_{\alpha}\right)$ be a substochastic Ray resolvent on $(K, \mathfrak{B}(K))$ with proper potential kernel. Furthermore, let $E \in \mathfrak{B}(K)$ be a subspace of the nonbranch points of $\left(W_{\alpha}\right)$ to which the resolvent may be restricted (i.e., $W_{\alpha}(x, K \backslash E)=0$ for all $x \in E$ and $\alpha>0$ ). Denote the restriction of $\left(W_{\alpha}\right)$ to $E$ by $\left(U_{\alpha}\right)$, and define $\mathfrak{E}:=E \cap \mathfrak{B}(K)$. Then $\left(U_{\alpha}\right)$ is the resolvent of a (transient) Borel right process on $(E, \mathfrak{E})$ if and only if the property (NSP) holds for $\left(U_{\alpha}\right)$.

Proof. The statement follows from (4.3) once one knows that $\left(U_{\alpha}\right)$ is a standard resolvent on $(E, \mathfrak{E})$. Since $\mathscr{S}(W) \cap \mathscr{E}(K)_{\mid E} \subset \mathscr{E}(U)$, and $\mathfrak{B}(K)=$ $\sigma(\mathscr{S}(W) \cap \mathscr{C}(K))$, the resolvent $\left(U_{\alpha}\right)$ is natural. Furthermore, since $E$ is a 
subspace of the nonbranch points of $\left(W_{\alpha}\right), 1 \in \mathscr{E}(U)$ and $\mathscr{E}(U)$ is $\wedge$-stable. Thus $\left(U_{\alpha}\right)$ is standard.

\section{EXISTENCE OF A RIGHT CONTINUOUS SEMIGROUP}

Again in this section the setup is the same as before.

(5.0) We assume we are given a resolvent $\left(U_{\alpha}\right)$ on a Lusin space $(E, \mathfrak{E})$ subject to hypotheses (3.0).

The set of nonbranch points for $\left(U_{\alpha}\right)$ (see Definition 3.1) will again be denoted by $N$. We are going to give a characterization of when there exists a right continuous semigroup on $(E, \mathbb{E})$ with resolvent $\left(U_{\alpha}\right)$ in terms of conditions on the $\left(U_{\alpha}\right)$-excessive measures. First, following Engelbert (see Chapter III, $\S 5$ of [7]) we give a general definition of a right continuous semigroup.

(5.1) Definition. Let $\left(P_{t}\right)_{t>0}$ be a semigroup of substochastic kernels on $(E, E)$. Let $\mathscr{R}$ denote the convex cone of all bounded $\left(P_{t}\right)$-supermedian functions $f$ for which $t \mapsto P_{t} f(x)$ is right continuous on the open interval ]0, $\infty$ [ for all $x \in E .\left(P_{t}\right)$ is called right continuous (on $] 0, \infty[$ ) if the cone $\mathscr{R}$ is $\wedge$-stable.

(5.2) Remarks. (1) Whenever there exists a right continuous semigroup with resolvent $\left(U_{\alpha}\right)$ it is unique, since Laplace transformation is one-one on right continuous functions.

(2) Let $\left(P_{t}\right)$ be a right continuous semigroup on $(E, \mathfrak{E})$ with resolvent $\left(U_{\alpha}\right)$. Then the following facts are immediate: Any $f \in \mathscr{R}$ satisfies $P_{t} f=P_{t} \hat{f}$ for $t>0$. The set $\Lambda b \mathscr{E}(U)$ of all finite infima of bounded excessive functions is contained in $\mathscr{R}$. Hence $\mathscr{R}$ forms a Ray cone for $\left(U_{\alpha}\right)$. Furthermore, $\mathscr{R}$ is closed under bounded increasing limits. In particular, in the representation for $N$ of (3.4.a), $N=\{\hat{g}=g\}, g$ is a function in $\mathscr{R}$ since it is a bounded increasing limit in $\Lambda b \mathscr{E}(U)$.

The following characterization of right continuity is due to Engelbert; cf. Theorem 16 in Chapter III, $\S 5$ of [7].

(5.3) Lemma. Let $\left(P_{t}\right)$ be a measurable semigroup on $(E, \mathfrak{E})$ with resolvent $\left(U_{\alpha}\right)$. The following properties are equivalent:

(i) $\left(P_{t}\right)$ is right continuous;

(ii) for all $f \in \Lambda \mathscr{E}(U)$ the function $t \mapsto P_{t}(x, f)$ is right continuous for any $x \in E$.

If $\left(U_{\alpha}\right)$ is the resolvent of a semigroup $\left(P_{t}\right)$ then since $\left(U_{\alpha}\right)$ is concentrated on $N$ (see 3.4) so are the kernels $P_{t}$ for almost all $t>0$. The next proposition shows that right continuity is characterized by the fact that all the kernels $P_{t}$ for $t>0$ are carried by $N$.

(5.4) Proposition. Let $\left(P_{t}\right)_{t>0}$ be a measurable semigroup on $(E, \mathfrak{E})$ with resolvent $\left(U_{\alpha}\right)$. Then $\left(P_{t}\right)$ is right continuous if and only if $P_{t}(x, E \backslash N)=0$ for all $t>0$ and all $x \in E$. 
Proof. Necessity follows from the remarks in (5.2.2) since $N=\{\hat{g}=g\}$ for some $g \in \mathscr{R}$ and $P_{t} g=P_{t} \hat{g}$ for $t>0$. Sufficiency is seen as follows: If $\left(P_{t}\right)$ is concentrated on $N$, then from the representation $N=\bigcap_{f \in \Lambda \mathscr{E}(U)}\{\hat{f}=f\}$ (see 3.4.a) we obtain $P_{t} f=P_{t} \hat{f}$ for all $t>0$ and all $f \in \Lambda \mathscr{E}(U)$; thus $\lim _{s \downarrow t} P_{s}(x, f)=\lim _{s \downarrow t} P_{s}(x, \hat{f})=P_{t}(x, \hat{f})=P_{t}(x, f)$ for any $x \in E$. Consequently, the right continuity follows from Engelbert's result (5.3).

Recall the definition of an entrance law $\left(\eta_{t}\right)_{t>0}$ with respect to a measurable semigroup $\left(P_{t}\right)_{t>0}$ on $(E, \mathfrak{E})$; i.e., $\left(\eta_{t}\right)_{t>0}$ is a family of $\sigma$-finite measures $\eta_{t} \in \mathscr{M}^{+}(E)$ such that $\eta_{s} P_{t}=\eta_{s+t}$ for all $s, t>0$. We are going to give a definition of an entrance law with respect to a resolvent, which is compatible with the above.

(5.5) Definition. A family $\left(\eta_{t}\right)_{t>0}$ of $\sigma$-finite measures $\eta_{t} \in \mathscr{M}^{+}(E)$ is called an entrance law with respect to $\left(U_{\alpha}\right)$ if $t \mapsto \eta_{t}(f)$ is Lebesgue measurable for any $f \in p \underline{\underline{\mathfrak{E}}}$ and if the following relation holds for all $\alpha \geq 0: \eta_{t} U_{\alpha}=$ $e^{\alpha t} \int_{t}^{\infty} e^{-\alpha s} \eta_{s} d s$

The following proposition shows that for right continuous semigroups $\left(P_{t}\right)$ the terms "entrance law w.r.t. $\left(P_{t}\right)$ " and "entrance law w.r.t. $\left(U_{\alpha}\right)$ " coincide under some finiteness condition.

(5.6) Proposition. Assume hypotheses (3.0). Let $\left(U_{\alpha}\right)$ be the resolvent of a right continuous semigroup $\left(P_{t}\right)$, and let $\left(\eta_{t}\right)_{t>0}$ be a family of $\sigma$-finite measures such that $t \mapsto \eta_{t}(f)$ is Lebesgue measurable for any $f \in p \underline{\underline{\mathfrak{E}}}$ and such that $\int_{0}^{\infty} \eta_{t} d t$ is $\sigma$-finite. Then $\left(\eta_{t}\right)$ is an entrance law with respect to $\left(P_{t}\right)$ if and only if $\left(\eta_{t}\right)$ is an entrance law with respect to $\left(U_{\alpha}\right)$ and there exists a Riesz cone $C \subset \mathscr{R}$ containing $b \mathscr{E}(U)$ such that $t \mapsto \eta_{t}(f)$ is right continuous for all $f \in C$ and such that for any $t>0$ there is an $h \in C, h>0$, satisfying $\eta_{t} U_{\alpha} h<\infty$ for all $\alpha>0$. In addition, if $\left(\eta_{t}\right)$ is an entrance law then it is concentrated on $N$.

Proof. $\Rightarrow$ : Set $C:=\mathscr{R}$. Then using $\eta_{t}=\eta_{s} P_{t-s}$ for $0<s<t$ we obtain the measurability in (5.5) by measurability of $\left(P_{t}\right)$, the right continuity of $t \mapsto \eta_{t}(f)$ for $f \in \mathscr{R}$ by definition of $\mathscr{R}$, and the additional statement that $\left(\eta_{t}\right)$ is concentrated on $N$ by (5.4). Furthermore, the integral equality for $\eta_{t} U_{\alpha}$ in (5.5) is immediate by Laplace transformation. Finally, let $m:=\int_{0}^{\infty} \eta_{t} d t$; then for $g>0$ with $m(g)<\infty$, the function $h:=U g$ satisfies $\eta_{t} U_{\alpha} h \leq m(g)<\infty$.

$\Leftarrow$ : For $s, t>0$ we obtain: $\int_{0}^{\infty} e^{-\alpha t} \eta_{s} P_{t} d t=\eta_{s} U_{\alpha}=e^{\alpha s} \int_{s}^{\infty} e^{-\alpha t} \eta_{t} d t=$ $\int_{0}^{\infty} e^{-\alpha t} \eta_{s+t} d t$; thus particularly for all $f \in C \wedge h$ the Laplace transforms of $\eta_{s} P_{t}(f)$ and $\eta_{s+t}(f)$ are finite and coincide. Since for $f \in C \wedge h$ both $t \mapsto$ $\eta_{s} P_{t}(f)$ and $t \mapsto \eta_{s+t}(f)$ are right continuous, the Laplace transformation is one-one for those functions, which implies $\eta_{s} P_{t}(f)=\eta_{s+t}(f)$ for all $f \in C \wedge h$, hence $\eta_{s} P_{t}=\eta_{s+t}$ since $C \wedge h-C \wedge h$ forms a Stone space.

We now come to the main result of this section, the characterization of the existence of a right continuous semigroup with resolvent $\left(U_{\alpha}\right)$. Recall that 
$m \in \mathscr{M}^{+}(E)$ is purely excessive (i.e., $\left.m \in \operatorname{Pur}(U)\right)$ if $m \in \operatorname{Exc}(U)$ and $\alpha m U_{\alpha}$ tends to zero as $\alpha \downarrow 0$.

(5.7) Theorem. Under hypotheses (3.0) there exists a (unique) right continuous semigroup $\left(P_{t}\right)_{t>0}$ with resolvent $\left(U_{\alpha}\right)$ if and only if the following condition (ENT) holds:

(ENT) $\left\{\begin{array}{l}\text { For any } m \in \operatorname{Pur}(U) \text { there exists a (unique) entrance law } \\ \left(\eta_{t}\right)_{t>0} \text { with respect to }\left(U_{\alpha}\right) \text { that represents } m \text { (i.e. } \\ \left.m=\int_{0}^{\infty} \eta_{t} d t\right) \text { and is concentrated on } N\left(\text { i.e., } \eta_{t}(E \backslash N)=0\right. \\ \text { for all } t>0) .\end{array}\right.$

Proof. Necessity of (ENT) is seen by the result [16] of Janssen mentioned in the introduction: $\left(P_{t}\right)$ is supposed to be right continuous, hence according to (5.4) $\left(P_{t}\right)$ is concentrated on $N$. Recalling the properties of $N$ stated in (3.4), one sees that the space $\left(N, \mathfrak{E}_{\mid N}\right)$ and the restrictions of $m,\left(U_{\alpha}\right)$, and $\left(P_{t}\right)$ to $N$ satisfy the hypotheses of Janssen's result (the corollary of Theorem 1 in [16])-in particular, $\left(N, \mathfrak{E}_{\mid N}\right)$ is Lusin and (UC) is satisfied w.r.t. $\left(U_{\alpha}\right)$ on $N$ according to (3.4). Hence there exists a (unique) entrance law $\left(\eta_{t}\right)$ w.r.t. $\left(U_{\alpha}\right)$ that represents $m$ and is concentrated on $N$.

To prove sufficiency of (ENT), for $x \in E$ consider the entrance law $\left(\eta_{t}^{x}\right)_{t>0}$ representing $\varepsilon_{x} U \in \operatorname{Exc}(U)$. We claim that $P_{t}(x):,=\eta_{t}^{x}$ for $x \in E$ and $t>0$ defines the desired semigroup. First, note that for potentials $U g \in \mathscr{E}$, it follows by definition (5.5) with $\alpha \downarrow 0$ that $\eta_{t}^{x}(U g)=\int_{t}^{\infty} \eta_{s}^{x}(g) d s \quad(*)$ holds. Therefore $\eta_{t}^{x}(U g) \leq \varepsilon_{x} U g=U g(x)$, and by choosing a sequence $\left(U g_{n}\right) \uparrow 1$ one obtains $\eta_{t}^{x} 1 \leq 1$ for all $t$ and $x$. Furthermore, the function $t \mapsto \eta_{t}^{x}(f)$ is right continuous and finite for all $f \in \Lambda b \mathscr{E}(U)$. This is immediate for bounded potentials $U g$ using relation (*) again, and it follows for all $f \in \Lambda b \mathscr{E}(U)$ by approximating $\hat{f}$ from below by bounded potentials (since $\left(\eta_{t}^{x}\right)$ is concentrated on $\{\hat{f}=f\}$-see 5.2.2). Therefore, by Lemma (3.12) of [11] we obtain the joint measurability of $(t, x) \mapsto \eta_{t}^{x}(f)$ for any $f \in \Lambda b \mathscr{E}(U)$. Since $\Lambda b \mathscr{E}(U)$ forms a Riesz cone, this implies the joint measurability of $(t, x) \mapsto \eta_{t}^{x}(f)$ for any $f \in$ $p \underline{\underline{E}}$. Furthermore, since for all $\alpha>0$ and all $x \in E$ the subsequent equalities hold, the resolvent equation implies the representation $\varepsilon_{x} U_{\alpha}=\int_{0}^{\infty} e^{-\alpha s} \eta_{s}^{x} d s$ :

$$
\begin{aligned}
\alpha \varepsilon_{x} U U_{\alpha} & =\alpha \int_{0}^{\infty} \eta_{t}^{x} U_{\alpha} d t=\alpha \int_{0}^{\infty} \int_{t}^{\infty} e^{\alpha t} e^{-\alpha s} \eta_{s}^{x} d s d t \\
& =\int_{0}^{\infty}\left(1-e^{-\alpha s}\right) \eta_{s}^{x} d s=\varepsilon_{x} U-\int_{0}^{\infty} e^{-\alpha s} \eta_{s}^{x} d s
\end{aligned}
$$

Hence, $\left(U_{\alpha}\right)$ forms the Laplace transform of the kernels $P_{t}, t>0$. The right continuity of $\left(P_{t}\right)$ follows as well from the right continuity of $t \mapsto P_{t} f(x)$ for any $x \in E$ and $f$ in the Riesz cone $\Lambda b \mathscr{E}(U)$. It remains to show the semigroup property: By the same argument as in the first part of the proof applied to the function $P_{s} f=P_{s} \hat{f}$ one sees that $t \mapsto \eta_{t}^{x}\left(P_{s} f\right)$ is right continuous for 
any $f \in \Lambda b \mathscr{E}(U)$ and any $s>0$. Therefore, by unicity of Laplace transforms, from the equalities

$$
\int_{0}^{\infty} e^{-\alpha s} \eta_{t}^{x}\left(P_{s} f\right) d s=\eta_{t}^{x}\left(U_{\alpha} f\right)=\int e^{-\alpha s} \eta_{s+t}^{x}(f) d s
$$

we conclude $\eta_{t}^{x}\left(P_{s} f\right)=\eta_{s+t}^{x}(f)$ for all $s, t>0$ and all $f \in \Lambda b \mathscr{E}(U)$; hence $\eta_{t}^{x} P_{s}=\eta_{s+t}^{x}$, which yields the semigroup property $P_{t} P_{s}=P_{s+t}$ for all $s, t>0$. Consequently, $\left(P_{t}\right)_{t>0}$ defined by $P_{t}(x):,=\eta_{t}^{x}$ for $t>0$ and $x \in E$ forms a substochastic right continuous semigroup with resolvent $\left(U_{\alpha}\right)$; this establishes the sufficiency of the statement (uniqueness is clear by 5.2.1).

Of course, by means of Theorems (4.1) and (5.7) it is obvious that properties (UPC) and (NSP) imply property (ENT), since any right process possesses a right continuous semigroup. A direct way of proving this can be obtained by reworking Fitzsimmons' proof in [9] in terms of the resolvent (in place of the semigroup).

\section{REMARKS ON GENERALIZATIONS}

This final section contains generalizations of the main results. We consider possible weakening of hypotheses (3.0), in particular the situation where the assumption " $U$ proper" is dropped. Finally, the relation of our definition of a nonbranch point to those of Engelbert and Wittmann is discussed.

Let us start with the situation (3.0) without the transience assumption:

(6.0) $\left(U_{\alpha}\right)_{\alpha>0}$ is a substochastic natural and normal resolvent on a Lusin space $(E, \mathfrak{E})$.

Then, naturally, our definition (3.1) of a nonbranch point for $\left(U_{\alpha}\right)$ no longer makes sense in general-as, e.g., an example like (1.7.3) suitably modified into a motion on a circle shows, since then there are no potentials except the zero measure. The proper definition, however, involves the $r$-potentials for $r>0$. Before stating it, we make a few preliminary remarks. To simplify notation let $\left(U_{\alpha}^{r}\right)$ denote the resolvent $\left(U_{r+\alpha}\right)_{\alpha>0}$ for $r \geq 0$. Then, of course, $\mathscr{E}^{r}(U)=$ $\mathscr{E}\left(U^{r}\right)$ and $\operatorname{Exc}^{r}(U)=\operatorname{Exc}\left(U^{r}\right)$, for $r \geq 0$.

(6.1) Remarks. (1) For any $r>s>0$ and any $\sigma$-finite measure $m \in \mathscr{M}^{+}(E)$ the following is true: $m \in \operatorname{Exc}^{s}(U)$ if and only if there exists a measure $m^{\prime} \in \operatorname{Exc}^{r}(U)$ such that $m=m^{\prime}\left(I+(r-s) U_{s}\right)$. If $m=\sup _{n} \mu_{n} U_{s}$ with $\mu_{n} U_{s} \in \operatorname{Pot}^{s}(U)$, then $m^{\prime}=\sup _{n} \mu_{n} U_{r}$ with $\mu_{n} U_{r} \in \operatorname{Pot}^{r}(U)$. If, moreover, the potential kernel $U$ is proper, then the statment holds for any $r>s \geq 0$.

(2) Remark (1) implies that each of the properties of excessive measures listed in (1.5) is satisfied with respect to the resolvent $\left(U_{\alpha}^{r}\right)$ if and only if it is satisfied with respect to $\left(U_{\alpha}^{s}\right.$ ), for any $r, s>0$ (resp., for any $r, s \geq 0$ provided $U$ is proper). To see this note that for $r>s>0$ with $(r-s)$ sufficiently small, the inverse operator of $\left(I+(r-s) U_{s}\right)$ exists and is given by $\sum_{n \in \mathbf{N}_{0}}\left((s-r) U_{s}\right)^{n}$; furthermore, in case $U$ is proper, recall from (2.3.1) that all properties in (1.5) 
are invariant under the transformation of (2.1), and the transform $\left(V_{\alpha}\right)$ has a bounded potential kernel such that for $r>s=0$ the same argument applies.

(3) Accordingly, each of the $\wedge$-stability properties of supermedian and excessive functions in (1.5) holds with respect to the resolvent $\left(U_{\alpha}^{r}\right)$ if and only if it holds with respect to $\left(U_{\alpha}^{s}\right)$, for any $r, s>0$ (resp., $r, s \geq 0$ provided $U$ is proper). We give the proof, e.g., for the property (IE). The argument applies whether $r>s$ or $s>r$ : If $\mathscr{E}^{r}$ is $\wedge$-stable and $f, g \in b \mathscr{E}^{s}$, then $f \wedge g \in \mathscr{S}^{s}$ and

$$
\begin{aligned}
\lim _{\alpha \rightarrow \infty} \alpha U_{s+\alpha}(f \wedge g) & =\lim _{\beta \rightarrow \infty} \beta U_{r+\beta}(f \wedge g)+(r-s) \cdot \lim _{\beta \rightarrow \infty} U_{r+\beta}(f \wedge g) \\
& =\lim _{\beta \rightarrow \infty} \beta U_{r+\beta}(f \wedge g)=f \wedge g,
\end{aligned}
$$

and the $\wedge$-stability of $b \mathscr{E}^{s}$ implies the $\wedge$-stability of $\mathscr{E}^{s}$.

Remark (6.1.2) implies that the subsequent definition for a nonbranch point extends Definition (3.1) to the situation defined in (6.0).

(6.2) Definition. We call a point $x \in E$ a nonbranch point for $\left(U_{\alpha}\right)$ if, for some $r>0, x$ is a nonbranch point for $\left(U_{\alpha}^{r}\right)$ in the sense of Definition (3.1).

Of course, as a consequence of (6.1.2), the term "some $r>0$ " in (6.2) may be replaced by "any $r>0$ ", and in case the potential kernel is proper, by "some (any) $r \geq 0$ ". Furthermore, according to Remarks (6.1), the results of $\S 3$ may be carried over to this situation to yield, among others, especially the following characterization.

(6.3) Corollary. Under hypotheses (6.0), $x \in E$ is a nonbranch point for $\left(U_{\alpha}\right)$ (in the sense of 6.2) if and only if one of the following equivalent conditions is satisfied:

(i) $\widehat{f \wedge} g(x)=f \wedge g(x)$ for any $f, g \in \mathscr{E}\left(U^{r}\right)$, for some (any) $r>0$;

(ii) the cone $\left\{f \in b \mathscr{S}^{r}(U): \hat{f}(x)=f(x)\right\}$ is $\wedge$-stable for some (any) $r>0$;

(iii) $\left(S S P_{x}\right)$ and $\left(U P C_{x}\right)$ hold for $\left(U_{\alpha}^{r}\right)$ for some (any) $r>0$.

Moreover, if the potential kernel is proper, " $r>0$ " may be replaced by " $r \geq 0$ " in each of statements (i) through (iii).

As well as the characterization of nonbranch points, the main results of $\S \S 4$ and 5 carry over to this more general situation:

(6.4) Corollary. Under hypotheses (6.0), there exists a Borel right process on $(E, \mathfrak{E})$ with resolvent $\left(U_{\alpha}\right)$ if and only if properties (UPC) and (NSP) are satisfied for the resolvent $\left(U_{\alpha}^{r}\right)$ for some (any) $r>0$.

Proof. Necessity is clear by applying Theorem (4.1) to the $r$-subprocess. As to the sufficiency, (4.1) provides a right process with resolvent $\left(U_{\alpha}^{r}\right)$ for some $r>0$, i.e., measures ${ }^{r} P^{x}$ on path space for $x \in E$. Consider the spacetime process associated with ${ }^{r} P^{x}$, which is a Borel right process on $(E \times \mathbf{R}$, $\mathfrak{E} \otimes \mathfrak{B}(\mathbf{R}))$. Let $h$ denote the function $(x, s) \mapsto \exp (r s)$ on $E \times \mathbf{R} ; h$ is easily seen to be excessive with respect to the space-time semigroup. Now the $h$ transform of the space-time process yields another Borel right process on $E \times \mathbf{R}$ 
(cf. XVI-28 of [4]), and its projection onto the first coordinate gives the desired Borel right process on $E$ with resolvent $\left(U_{\alpha}\right)$, as is readily checked. The reason for this lies in the particular form of the function $h$, which is independent of the first variable and is such that it compensates the "killing at rate $r$ ".

(6.5) Corollary. Under hypotheses (6.0), there exists a (unique) right continuous semigroup $\left(P_{t}\right)$ with resolvent $\left(U_{\alpha}\right)$ if and only if the condition (ENT) (see 5.7) holds for the resolvent $\left(U_{\alpha}^{r}\right)$ for some (any) $r>0$.

Proof. Necessity as in the proof of (6.4); sufficiency similarly: Theorem (5.7) provides a right continuous semigroup $\left(P_{t}^{r}\right)$ with resolvent $\left(U_{\alpha}^{r}\right)$ for some $r>$ 0 ; it is easily seen that $P_{t}:=e^{r t} P_{t}^{r}$ for $t>0$ is independent of $r>0$ and thus defines the desired right continuous semigroup $\left(P_{t}\right)$ with resolvent $\left(U_{\alpha}\right)$.

So far we have seen that the assumption on the potential kernel to be proper in the previous sections only simplified the presentation. What remains to be discussed are the assumptions on the underlying space $(E, \mathfrak{E})$ and on the relation of $\mathbb{E}$ with the $\left(U_{\alpha}\right)$-excessive functions. For the results of $\S 3$ the assumption $(E, \mathfrak{E}) \quad U$-space (cf. [11]) instead of Lusin would suffice; $(E, \mathfrak{E}) \quad U$-space is needed in the proof of $(\mathrm{a}) \Rightarrow(\mathrm{b} 1) \Rightarrow(\mathrm{c})$ in Theorem (3.3), because some measurability of $E$ in the Ray compactification is required. The implication (a) $\Rightarrow(\mathrm{bl})$ is used moreover in the proofs of Theorems (3.5) and (4.1). As Fitzsimmons pointed out to me, there is a direct way of proving (3.5) without using the Ray compactification. The proof of the main result in $\S 4$, Theorem (4.1), needs some measurability of $E$ in the Ray compactification that is "better than universally measurable", since the state space of a right process is nearly Ray Borel (see, e.g., [17]). An example pointed out to me by T. S. Salisbury (cf. [20]) shows that Theorem (4.1) with $(E, \mathfrak{E})$ a $U$-space instead of Lusin is not right. As in $\S 3$, the main result in $\S 5$, Theorem $(5.7)$, only requires $(E, \mathfrak{E})$ to be a $U$-space, as does Janssen's result that is used in the proof of (5.7). Here, moreover, the assumption " $\left(U_{\alpha}\right)$ natural and normal" may be replaced by the weaker hypothesis " $\mathfrak{E}=\mathfrak{E}^{\prime}$ (as defined in 2.3)". The reason is that the proof of this more general statement may be reduced to the situation of (5.7) by applying a Doob transformation to the semigroup and entrance law in question.

Recall the notion of the transformed resolvent $\left(V_{\alpha}\right)$ from $(2.1)$, the definition of $\mathfrak{E}^{\prime}$ from (2.3), and the remarks below (2.3).

As to the results on nonbranch points, the most general situation in which some characterization as in Theorem (3.3) is still valid is the following:

(6.6) $\left(U_{\alpha}\right)$ is a substochastic nondegenerate resolvent on a measurable space $(E, \mathfrak{E})$, where $\mathfrak{E}$ is generated by the class of quotients of measurable $r$-excessive functions for some (all) $r>0$.

For reasons of simplicity, however, we state the subsequent results again under the additional assumption that $U$ is proper; then the condition on the $\sigma$-algebra is equivalent with “ $\mathfrak{E}=\mathfrak{E}^{\prime}$. ." 
(6.7) Corollary. Assume the situation of (6.6) and suppose in addition that the potential kernel $U$ is proper. Then for $x \in E$ the properties $\left(\mathrm{EPC}_{x}\right),\left(\mathrm{IE}_{x}\right)$, and $\left(\mathrm{IS}_{x}\right)$ are equivalent among each other and with the following properties ( with respect to the resolvent $\left(V_{\alpha}\right)$ ):

$$
\begin{gathered}
\widehat{f \cdot g}(x)=f(x) \cdot g(x) \quad \text { for all } f, g \in \mathscr{E}(V) ; \\
\widehat{f \cdot g}(x)=\hat{f}(x) \cdot \hat{g}(x) \quad \text { for all } f, g \in \mathscr{S}(V) ; \\
\{f \in b \mathscr{S}(V)-b \mathscr{S}(V): \hat{f}(x)=f(x)\} \quad \text { is product stable. }
\end{gathered}
$$

If, in addition, $1 \in \mathscr{E}(U)$, then one may as well replace $\left(V_{\alpha}\right)$ by $\left(U_{\alpha}\right)$ in $(*)-$ $(* * *)$.

Proof. Under the given hypotheses, $\left(V_{\alpha}\right)$ becomes a natural and normal resolvent on $(E, \mathfrak{E})$ with proper potential kernel $V$ satisfying $V 1=1$. In particular-aside from the assumption that $(E, \mathfrak{E})$ is Lusin- $\left(V_{\alpha}\right)$ satisfies hypotheses (3.0). An investigation of the proof of Theorem (3.3) shows that the assumption $(E, \mathfrak{E})$ Lusin is used only in connection with the Ray compactification, i.e., in the part $(\mathrm{a}) \Rightarrow(\mathrm{b} 1) \Rightarrow(\mathrm{c})$. Hence the proof of $(3.3)$ with Fitzsimmons's version of $(\mathrm{a}) \Rightarrow(\mathrm{c})$ via (b2) shows the equivalence of $\left(\mathrm{EPC}_{x}\right)$, $\left(\mathrm{IE}_{x}\right),\left(\mathrm{IS}_{x}\right)$, and $(*)$ with respect to $\left(V_{\alpha}\right)$. The equivalence of $(* *)$ and $(* * *)$ with $(*)$ is immediate. Since the first three properties are invariant under the transformation of $\left(U_{\alpha}\right)$ to $\left(V_{\alpha}\right)$, the first part is established. The second part follows from the fact that, if $1 \in \mathscr{E}(U)$ then property $(*)$ is invariant under the transformation of $\left(U_{\alpha}\right)$ to $\left(V_{\alpha}\right)$ in $(2.1)$, which is easily seen from $(2.1)$ using $(U q)^{-1} \in \mathscr{E}(V)$.

(6.8) Corollary. Assume the situation of (6.6) and suppose in addition that the potential kernel $U$ is proper. Then properties (EPC), (IE), and (SSP) together with (UPC) or (UC) are equivalent among each other and with the property that $b \mathscr{E}(V)-b \mathscr{E}(V)$ forms an algebra. If, in addition, $1 \in \mathscr{E}(U)$, then the statement that $b \mathscr{E}(U)-b \mathscr{E}(U)$ forms an algebra is equivalent as well.

Proof. The statement follows as in (6.7) from the proof of (3.6).

We conclude this section with a few remarks on the definitions of nonbranch points by Engelbert and Wittmann.

In Chapter III, $\S 4$ of [7], Engelbert defines a nonbranch point for a natural and normal resolvent as a point at which all $r$-excessive functions $(r \geq 0)$ are essentially stochastically continuous (cf. Definition 18 of $I, \S 1$ of [7]). In case of a proper potential kernel this definition is equivalent with the statement just for $r=0$.

In Chapter I, $\S 3$ of [22], Wittmann gives a definition of a nonbranch point in a completely general context, but which in case of a normal and natural resolvent coincides with Engelbert's (as proved in Korollar 17 of Chapter II, $\S 4$ of [22]).

Wittmann proves furthermore (cf. Korollar 20 of I, $\S 3$ of [22]) that a point $x \in E$ is a nonbranch point in his sense if and only if property (i) of (6.3) 
holds. Therefore, under hypotheses (6.6) our definition (6.2) of a nonbranch point coincides with Wittmann's and, specialized to the situation of [7], as well with Engelbert's.

Wittmann's investigations show, furthermore, that Engelbert's characterization of a nonbranch point as given in Theorem 3(1)-(2) of III, $\S 4$ of [7] does not require the existence of an associated Markov process, and that Theorem 29 of I, $\S 2$ of [7] can be improved. This, together with our preceding results, entails the following final corollary.

(6.9) Corollary. In the context of Engelbert [7] (i.e., $\left(U_{\alpha}\right)$ is a natural and normal resolvent on a measurable space $(E, \mathfrak{E}))$, the following properties are always equivalent:

(1) $x$ is a nonbranch point in the sense of [7];

(2) the cone $\left\{f \in \mathscr{S}^{r}(U): \hat{f}(x)=f(x)\right\}$ is $\wedge$-stable for all $r \geq 0$;

(3) $\lim _{\alpha} \alpha U_{\alpha}(x,|f-f(x)|)=0$ for all $f \in \mathscr{S}^{r}(U)$ satisfying $f(x)<\infty$ and for all $r \geq 0$;

(4) any $f \in \mathscr{S}^{r}(U)$ is essentially stochastically continuous at $x$ for all $r \geq 0$.

(5) $x$ is a nonbranch point in the sense of definition (6.2);

(6) each of the properties appearing in the statement of (6.7) holds with respect to the resolvent $\left(U_{\alpha}^{r}\right)$ for all $r>0$.

Proof. (a) (1) $\Rightarrow(2) \Rightarrow(3)$ and (4) $\Rightarrow(1)$ are proved by Engelbert (see III, $\S 4$ of [7]).

(3) $\Rightarrow$ (4) follows from S.12 and L.13 of I, $\S 4$ of [22], which proves (4) for any $f \in \mathscr{S}^{r}(U)$ finite at $x$.

$(2) \Leftrightarrow(5) \Leftrightarrow(6)$ follows by (6.7).

\section{REFERENCES}

1. H. Bauer, Wahrscheinlichkeitstheorie und Grundzüge der Masstheorie, 2nd ed., De Gruyter, Berlin and New York, 1974.

2. R. M. Blumenthal and R. K. Getoor, Markov processes and potential theory, Academic Press, New York, 1968.

3. C. Dellacherie and P. A. Meyer, Probabilité et potentiel, Hermann, Paris, 1975, Chapters I-IV.

4. __ Probabilité et potentiel, Hermann, Paris, 1987, Chapters XII-XVI.

5. E. B. Dynkin, Markov processes, vol. I, Springer, Berlin, 1965.

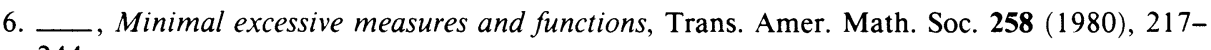
244.

7. H. J. Engelbert, Markov processes in general state spaces, Part I, Math. Nachr. 80 (1977), 19-36; Part II, Math. Nachr. 82 (1978), 191-203; Part III, Math. Nachr. 83 (1978), 47-71; Part IV, Math. Nachr. 84 (1978), 277-300; Part V, Math. Nachr. 85 (1978), 111-130; Part VI, Math. Nachr. 85 (1978), 235-266; Part VII, Math. Nachr. 86 (1978), 67-83.

8. __ On the construction of semigroups from substochastic resolvents, Math. Nachr. 105 (1982), 171-192.

9. P. J. Fitzsimmons, On two results in the potential theory of excessive measures, preprint, 1986.

10. P. J. Fitzsimmons and B. Maisonneuve, Excessive measures and Markov processes with random birth and death, Probab. Theory Related Fields 72 (1986), 319-336. 
11. R. K. Getoor, Markov processes: Ray processes and right processes, Lecture Notes in Math., vol. 440, Springer-Verlag, Berlin, Heidelberg, and New York, 1975.

12. R. K. Getoor and J. Glover, Markov processes with identical excessive measures, Math. Z. 184 (1983), 287-300.

13. __ Riesz decompositions in Markov process theory, Trans. Amer. Math. Soc. 185 (1984), 107-132.

14. __ Constructing Markov processes with random times of birth and death, Sem. Stochastic Processes, 1986, Birkhäuser, Boston, Mass., 1987, pp. 35-69.

15. W. Hansen, Konstruktion von Halbgruppen und Markoffschen Prozessen, Invent. Math. 3 (1967), 179-214.

16. K. Janssen, Representation of excessive measures, Sem. Stochastic Processes, 1986, Birkhäuser, Boston, Mass., 1987, pp. 85-105.

17. J. F. Mertens, Processus de Ray et théorie du balayage, Invent. Math. 23 (1974), 117-133.

18. P. A. Meyer, Le schéma de remplissage en temps continu d'après H. Rost, Séminaire Probabilité VI, Springer-Verlag, 1972, pp. 130-150.

19. D. Ray, Resolvents, transition functions and strongly Markovian processes, Ann. of Math. 70 (1959), 43-75.

20. T. S. Salisbury, Three problems from the theory of right processes, Ann. Probab. 15 (1987), 263-267.

21. M. J. Sharpe, The general theory of Markov processes (forthcoming).

22. R. Wittmann, Kac'sche Potentialtheorie für Resolventen, Markoffsche Prozesse und harmonische Räume, Verlag der Bayerischen Akademie der Wissenschaften, München, 1982.

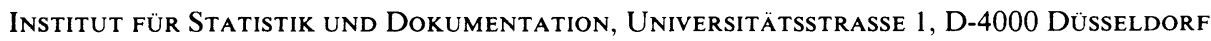
1, West Germany 